\title{
Do post-stroke patients benefit from robotic verticalization? A pilot-study focusing on a novel neurophysiological approach
}

\author{
Rocco Salvatore Calabrò ${ }^{*, 1}$, Antonino Naro ${ }^{1}$, Margherita Russo, Antonino Leo, Tina Balletta, \\ Ileana Saccà, Rosaria De Luca and Placido Bramanti \\ IRCCS Centro Neurolesi “Bonino-Pulejo”, Messina, Italy
}

\begin{abstract}
.
Background: Tilt-table equipped with the dynamic foot-support (ERIGO) and the functional electric stimulation could be a safe and suitable device for stabilization of vital signs, increasing patient's motivation for further recovery, decreasing the duration of hospitalization, and accelerating the adaptation to vertical posture in bedridden patients with brain-injury. Moreover, it is conceivable that verticalization may improve cognitive functions, and induce plastic changes at sensory motor and vestibular system level that may in turn facilitate motor functional recovery.

Objective: To test the safety and effectiveness of ERIGO treatment on motor and cognitive functions, cortical plasticity within vestibular and sensory-motor systems in a bedridden post-stroke sample.

Methods: 20 patients were randomly divided in two groups that performed ERIGO training (30 sessions) (G1) or physiotherapistassisted verticalization training (same duration) (G2), beyond conventional neurorehabilitation treatment. Motor and cognitive functions as well as sensory-motor and vestibular system plasticity were investigated either before $\left(\mathrm{T}_{0}\right)$ or after $\left(\mathrm{T}_{1}\right)$ the rehabilitative protocols.

Results: Both the verticalization treatments were well-tolerated. Notably, the G1 patients had a significant improvement in cognitive function $(p=0.03)$, global motor function $(p=0.006)$, sensory-motor $(p<0.001)$ and vestibular system plasticity $(p=0.02)$ as compared to $\mathrm{G} 2$.

Conclusions: ERIGO training could be a valuable tool for the adaptation to the vertical position with a better global function improvement, as also suggested by the sensory-motor and vestibular system plasticity induction.
\end{abstract}

Keywords: ERIGO, verticalization, stroke, vestibular system, sensory-motor plasticity, bedridden condition

\section{Introduction}

Stroke is the main cause of adult long-term disability, frequently leading to significant gait and trunk control impairment (Verheyden et al., 2005).

\footnotetext{
${ }^{1}$ These authors equally contributed to the work.

*Corresponding author: Rocco Salvatore Calabrò, IRCCS Centro Neurolesi "Bonino-Pulejo" Messina, S.S. 113, Contrada Casazza, 98124, Messina, Italy. Tel.: +39 09060128954; Fax: +390906012 8950; E-mail: salbro77@ tiscali.it.
}

Post-stroke sequelae, including limb paralysis or plegia, loss of line, agnosia, patient limited cooperation, deep sensory disorder, and/or ataxia as well as circulatory instability may lead to a bedridden condition. Patients who are unable to safely stand alone and perform independently transfers are at an increased risk for multiple medical complications (Gordon et al., 2004).

Thus, early mobilization and postural changes may be useful in improving physical and psychological outcomes. Indeed, physical therapy is essential in 
preventing contractures and pressure ulcers, and in stabilizing vital signs (blood pressure, heart rate, and ventilation), decreasing therefore the duration of bedridden and hospitalization, and increasing patients' motivation to further recover. Moreover, passive/active mobilization and verticalization procedures (VT) are the preliminary steps to the ambulation training.

Interestingly, it has been suggested that VT may play a role in stimulating cortical areas involved in trunk and lower limb control, so that deafferentation and learned non-use can be contrasted (Pittaccio et al., 2013). VT may actively contribute to enhance cognitive performances through an increase in cerebral blood flow with a consequent induction of cortical plasticity, especially in frontal lobes (Reinstrup et al., 1994). Another important issue to take into account is to what extent the vestibular system may play a role in the improvement of sensing self-motion and eyes and body stabilization in space, during and after VT (Cullen and Roy, 2004; Precht, 1979). Vestibular system plasticity improvement could promote the adaptation and the maintenance of the vertical position, and lead to behavioral changes aimed at minimizing the risk of hesitation and falling, by adopting a more cautious and stable stance, and enhancing several postural reflexes. In addition, it has been reported that vestibular system may influence the performance of some neuropsychological tests and, indirectly, sensory-motor system functionality (Andersonn et al., 2003; Risey and Briner, 1990; Yardley et al., 2001; Vallar et al., 1990, 1993).

Although physical therapy may be beneficial in limiting the effects of bedridden condition and stroke medical complications (so to facilitate maximal functional recovery), mobilizing severely impaired or non-cooperating patients is often unprofitable. In order to improve and standardize physiotherapy-assisted VT (pVT), several robotic devices have been developed. Indeed, it has been shown that robot-based rehabilitation improves motor performance by boosting brain plasticity, which plays a crucial role for motor control recovery, especially in stroke patients (Pellegrino et al., 2012; Duret et al., 2014; Basteris et al., 2014). In particular, the ERIGO device (Hocoma AG, Volketswil, Switzerland) combines progressive verticalization, cyclic leg movement (which allows stepping reinforcement in combination with step synchronized muscle functional electrical stimulation -FES- at lower limb), and body weight loading to ensure the safe stabilization of the patient in the upright position. Notably, FES stepping has been shown to be an effective tool for muscle strengthening and walking improvement in neurological patients, including hemiplegic ones (Vitenzon et al., 2005; Bogataj et al., 1995; Tong et al., 2006; Yan et al., 2005; Ferrante et al., 2008; Popovic et al., 2009). Robotic verticalization (rVT) shows many advantages in comparison to $\mathrm{pVT}$, including the body weight loading and the possibility to continuously perform cyclic leg movements, especially when coupled with FES. More in detail, rVT maximizes the potential for longitudinal weight bearing through the lower extremities in a position of hip-extension, knee-extension, and ankle-dorsiflexion, with exercises of body weight shifting from one leg to the other. Such training cannot be easily performed in severe post-stroke patients by using conventional physiotherapy (Peszczynski et al., 1972). Nevertheless, the presence of the physiotherapist is always essential (even in robotic neurorehabilitation), since patient's cooperation has to be constantly ensured and stimulated (Chang et al., 2013).

\subsection{Rationale and aims}

Even though the positive effects of the early VT are well-known (Baltz et al., 2013; Kuznetsov et al., 2013), the use of rVT in bedridden post-stroke patients in the sub-acute phase has received less attention. Therefore, aim of the study was to evaluate the effects of an rVT protocol on motor and cognitive performances, as well as on vestibular system and sensory-motor areas plasticity, in post-stroke bedridden patients in comparison to a standard $\mathrm{pVT}$.

\subsection{Objective}

To compare the effects of the rVT and pVT in two groups of post-stroke patients in the sub-acute phase. The primary outcomes consisted in the assessment of:

- vertical posture tolerance (the ability to sustain the tilt-table inclination without signs or symptoms of intolerance) and, thus, the rVT safety. This was evaluated in terms significant variations (set at $\pm 15 \%$ ) of vital parameters, i.e. mean blood pressure -MBP-, mean heart rate-HR-, mean oxygen saturation $-\mathrm{pO}_{2-}$, and fatigue and discomfort level during the verticalization, rated on a visual analogic scale (VAS, set at $\geq 7$ ). 
Table 1

Shows the clinical-demographic characteristics at baseline, which were non-significantly different $(p>0.2)$. Values are reported as mean \pm sd

\begin{tabular}{|c|c|c|c|}
\hline & & G1 & $\mathrm{G} 2$ \\
\hline \multirow[t]{8}{*}{ clinical-demographic characteristics } & Subjects (n) & 10 & 10 \\
\hline & Female (n) & 6 & 5 \\
\hline & Age (yy) & $71 \pm 3$ & $70 \pm 5$ \\
\hline & BMI & $26 \pm 2$ & $28 \pm 3$ \\
\hline & Hypertension (n) & 5 & 6 \\
\hline & DM type II (n) & 3 & 2 \\
\hline & Smokers (n) & 2 & 3 \\
\hline & Cardiac arrhythmia (n) & 2 & 1 \\
\hline Cognitive domain & $\mathrm{RCPM}$ & $17 \pm 3$ & $19 \pm 6$ \\
\hline \multirow{3}{*}{ Verticalization tolerance } & $\mathrm{BP}(\mathrm{mmHg})$ & $113 \pm 3$ & $116 \pm 6$ \\
\hline & $\mathrm{HR}(\mathrm{bpm})$ & $81 \pm 5$ & $90 \pm 6$ \\
\hline & $\mathrm{pO}_{2}(\%)$ & $98 \pm 2$ & $97 \pm 5$ \\
\hline \multirow[t]{3}{*}{ Clinical parameters } & MRC & $2 \pm 1$ & $2 \pm 3$ \\
\hline & FM & $13 \pm 3$ & $12 \pm 6$ \\
\hline & PASS & $3 \pm 1$ & $3 \pm 3$ \\
\hline \multirow[t]{3}{*}{ Electrophysiological parameters } & post-PAS MEP (mV) & $0.5 \pm 0.3$ & $0.5 \pm 0.4$ \\
\hline & $\operatorname{cVEP}(\mu \mathrm{V})$ & $2 \pm 0.2$ & $2 \pm 0.3$ \\
\hline & cVEMPS $(\mu \mathrm{V})$ & $12 \pm 2$ & $16 \pm 5$ \\
\hline
\end{tabular}

BMI: body mass index; DM: diabetes mellitus; RCPM: raven colored progressive matrices; BP: blood pressure; HR: heart rate; MRC: muscle research council; FM: Fugl-Meyer; PASS: Postural Assessment Scale for Stroke patients; cVEP: cortical vestibular evoked potentials; cVEMPs: cervical vestibular evoked myogenic potentials; PAS paired associative stimulation; MEP: motor evoked potential; n: number.

- effects on cognitive function (as per Ravens Coloured Progressive Matrices test, RCPM) and on the lower limbs functionality (Fugl-Meyer scale for lower limbs -FM-, Muscle Research Council -MRC).

- posture maintaining and changing (Postural Assessment Scale for Stroke patients, PASS).

As secondary outcome we considered the VT effects on some electrophysiological parameters (cortical vestibular evoked potentials -cVEP-, cervical vestibular evoked myogenic potentials -cVEMPs-, and sensory-motor plasticity by means of a paired associative stimulation -PAS- protocol).

\section{Methods}

\subsection{Participants and study design}

This preliminary study has been designed as a single centre, randomized, controlled trial. Of the 83 patients attending our in-patient Neurorehabilitation Unit of the IRCCS Centro Neurolesi Bonino-Pulejo, we selected a group of bedridden patients affected by ischemic stroke in the subacute phase (i.e. after 4 to 8 weeks from the stroke onset). All the 32 screened individuals had a stroke in the territory of the middle cerebral artery with a National Institutes of Health Stroke Scale score $\leq 20$. As exclusion criteria we considered: blood pressure (BP) higher than 220/120 mmHg or lower than $90 / 60 \mathrm{mmHg}$; internal carotid artery stenosis $>60 \%$ (Hathout et al., 2005); severe cardiac diseases; severe medical conditions; contractures of lower extremities; lower extremity thrombophlebitis or deep vein thrombosis; severe joint or bone pathologies; cortical excitability modifying drug intake; safety contraindication to transcranial magnetic stimulation (TMS); spinal cord and peripheral nervous system pathologies. Hence, we selected 20 patients, who thus underwent an intensive VT rehabilitation and were randomly allocated in the experimental $(\mathrm{G} 1, \mathrm{rVT})$ or the control group $(\mathrm{G} 2, \mathrm{pVT})$, through a random number table. The clinical-demographic characteristics are reported in Table 1. We are planning a larger-sample study with matched pairs of experimental groups. Baseline data indicate that the inter-group difference is normally distributed, with standard deviation of 0.2 . If the inter-group mean difference is $\geq 20 \%$, we will need to enroll 68 subjects per group in order to reject the null hypothesis (i.e. the response difference is zero with probability -power- of 0.9). The Type I error probability associated with this test of this null hypothesis is 0.05 .

\subsection{Interventions}

All participants practiced 45 minutes of standard physical treatment program, followed by VT after a 
resting period of 20 minutes with $30^{\circ}$ of head elevation. The 10 participants in G1 underwent rVT by means of the ERIGO+FES (Fig. 1), whereas those in G2 performed $\mathrm{pVT}$.

Motor and cognitive function, as well as sensorymotor and vestibular system plasticity, were investigated either before $\left(T_{0}\right)$ or after $\left(T_{1}\right)$ the rehabilitative protocols. The experimenters who applied the clinicalelectrophysiological tests and analyzed the data were blinded to patient's group identity. We employed at least 60' in order to perform the clinicalelectrophysiological evaluations that were carried-out after the standard physical treatment and before the VT.

\subsection{Standard physical treatment program}

Passive and active joint mobilization, muscle stretching, proprioceptive exercises, and postural and trunk stabilization were practiced at patient's bed for 45', according to standard procedures in physiotherapy setting (Peszczynski et al., 1972; Van der Wees and Mead, 2004; American Physical Therapy Association, 1997; Pollock et al., 2014; Young and Forster, 2007).

\section{4. rVT treatment (group G1)}

Each G1 participant underwent 30 sessions of rVT, using the robotic tilt-table ERIGO. It consists of a stretcher that can be tilted from zero to 90 degrees, with feet plates for leg loading and stepping-like movements. Indeed, training on the ERIGO combines mobilization out of bed, body verticalization, and rhythmic leg movement with cyclic loading. After the positioning and fastening over ERIGO device, G1 patients received a 30 minute daily training, 5 days per week (from Monday to Friday), for 6 weeks. During the first three training sessions, patients were gradually verticalized from 10 to $30^{\circ}$ over 15 minutes at the rate of $3^{\circ}$ in 5 seconds, and stepping was performed at a rate of 30 steps per minute. Loading of the legs was either passive or passive-active. By session 5 , verticalization was increased to $60^{\circ}$ over 15 minutes, and stepping to 35 steps per minute. By session 10 , verticalization reached $90^{\circ}$ with 40 steps per minute. If any sign of intolerance (vital signs, continuous requests of protocol withdrawal) was observed at any angle of inclination, the protocol was modified and participants got back to the previous position at the rate of $3^{\circ}$ per second. Thus, the participants continued the protocol, or in case of persistent intolerance they got back to a supine position. During the verticalization, each patient received a FES treatment using a six-channel stimulator (Motionstim-8, Medel GmbH, Hamburg, Germany). Electrodes were placed over biceps femoris, quadriceps femoris, tibialis anterior, and gastrocnemius. The stimulation was synchronized with robotic legs' movements: biceps femoris, and gastrocnemius muscles at leg flexion, quadriceps and tibialis anterior at leg extension. Stimulation intensity was set at $30 \mathrm{~mA}$, with a shift of $\pm 10 \mathrm{~mA}$ in order to avoid habituation.

\section{5. pVT treatment (group G2)}

Patients were treated for at least 15 minutes based on Bobath' method involving intensive preparatory training for verticalization in the sitting and standing positions (Hummelsheim and Mauritz, 1993), and with the assistance of a simple tilt-table for further 15 minutes. Elevation activities started under supervision. The patient reached the sitting position in bed through a trapeze or a half side rail. Since patients were hemiplegic and thus tended to lean toward the involved side when sitting, balance training with over-correction toward the opposite side was performed. Once seated in bed, the patient was upgraded to sitting on the bed-side, followed by the standing/standing-transfer procedures and, therefore, the bed-to-chair and vice-versa transfers. After such stages, the patient started pVT by means of a simple tilt-table, with the verticalization procedures similar to the rVT. Standard balancing exercises requiring a shift of body weight alternately from one leg to the other, in order to maintain independently the upright position, were also performed.

\subsection{Clinical parameters}

After the standard physical treatment program, we measured the MBP for 10 minutes, according to the $\mathrm{MBP}=\mathrm{DP}+(\mathrm{PP} / 3)$ formula (where DP is diastolic pressure and $\mathrm{PP}$ is the pulse pressure, i.e. the difference between the systolic and diastolic pressure), the mean $\mathrm{HR}$ within 10 minutes, the mean $\mathrm{pO}_{2}$ for 10 minutes while in room-air. Such parameters were collected by means of CMS9000 Patient Monitor (Contecmed, Qinhuangdao, Hebei Provice, PRC). During each VT session, we also measured the perceived exertion and the requests to discontinue the treatment, by means 


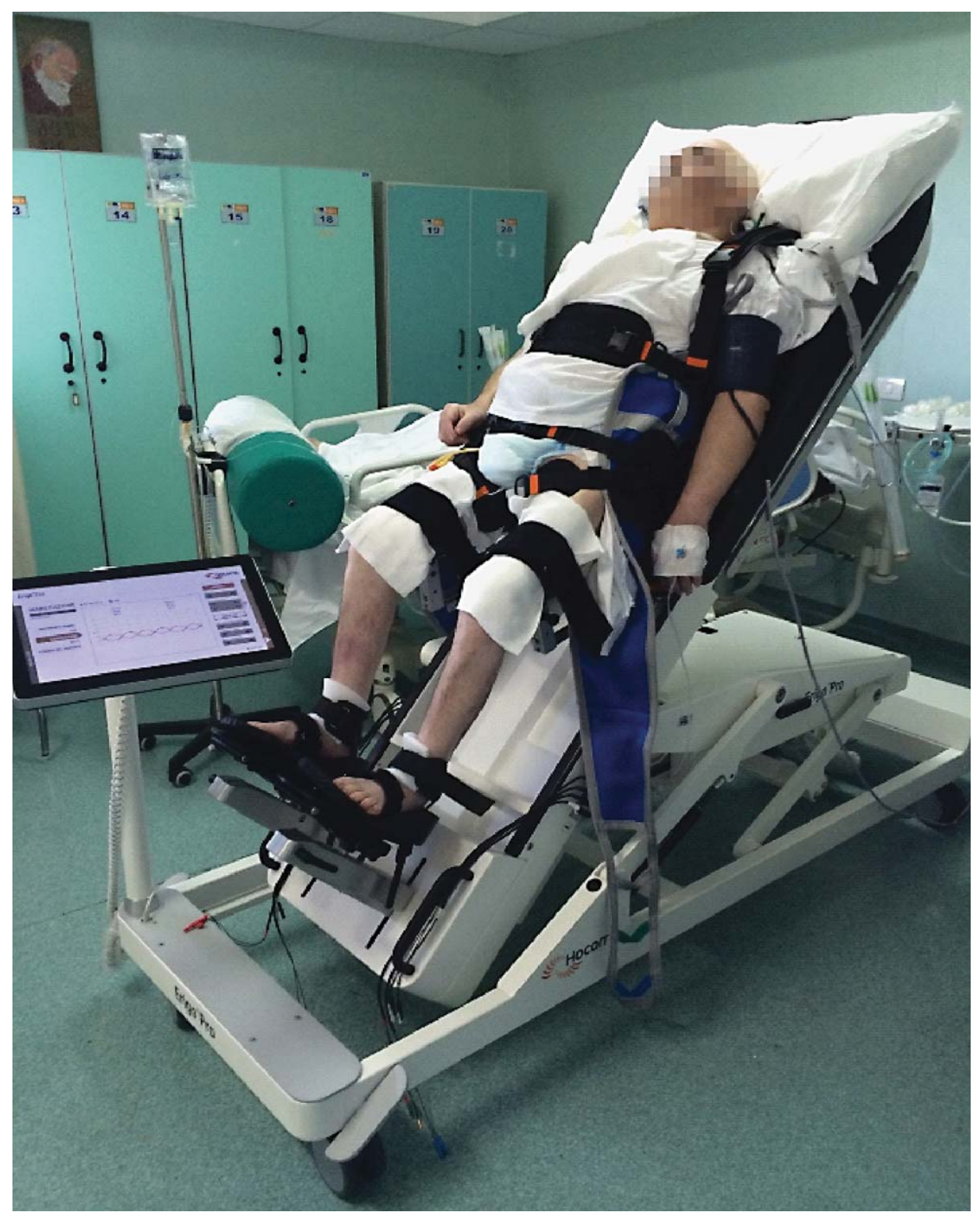

Fig. 1. ERIGO device combines progressive verticalization, cyclic leg movements in combination with step synchronized muscle functional electric stimulation at lower limb (that allow stepping reinforcement), and body weight loading, in an attempt to ensure the safe stabilization in the upright position of the patient.

of a VAS, beyond continuous vital sign monitoring. The lower limb motor function was evaluated through the FM (Gladstone et al., 2002). The paresis severity of lower limb was evaluated through the MRC scale (hip flexion, knee extension, and ankle dorsiflexion) (Medical Research Council, 1981). We also monitored the patient's postural control by means of the PASS scale (Benaim et al., 1999).

\subsection{Cognitive abilities}

RCPM is a non-verbal assessment tool, consisting in multiple choice questions, listed in order of difficulty, and designed to measure an individual's ability to perceive and think clearly, make meaning-out of confusion and formulate new concepts when dealing with novel information. Such issues constitute the so-called 
general intelligence. The colored version we used in our work has been designed for mentally and physically impaired individuals. In each item, the subjects were asked to identify the missing element that completes a pattern (Raven et al., 2004).

\subsection{Electrophysiological parameters: cVEMPs}

cVEMPs protocol has been formerly defined (Colebatch et al., 1994). cVEMPs assess the vestibular function through the Vestibulo-Collic reflexes, which act on the neck muscles in order to stabilize the head. The VCR neural circuitry may include saccule, inferior vestibular nerve, vestibular nuclear complex, medial vestibulospinal tract, the spinal accessory nerve transmitting signals to the sternocleidomastoid muscle. Different stimuli have been used to evoke cVEMPs. In particular, the galvanic stimulation (short-duration transmastoid direct current stimulation) could activate irregularly-firing afferents and a vestibulo-collic pathway, by-passing the vestibular end-organ. The cathodal stimulation results in a firing increase whereas the anodal in a decrease (Goldberg et al., 1984; Kim et al., 2004). A response consisting of a biphasic positivenegativity wave, peaking at 13 and $23 \mathrm{~ms}$ respectively (P13-N23), can be registered from the sternocleidomastoid muscle in normal subjects (Zhou and Cox, 2004; Rosengren et al., 2011; Uchino et al., 1999). We applied a cathodal current of $4 \mathrm{~mA}$ for $1 \mathrm{~ms}$ at $5 \mathrm{~Hz}$ on the mastoid of the affected side (the reference electrode was placed over the contralateral mastoid). EMG activity was recorded through $\mathrm{Ag}-\mathrm{AgCl}$ surface electrodes applied to both the sternocleidomastoid muscles using a classic muscle belly-tendon montage. Ground electrode was put on sternum. Signals were amplified and filtered (from $5 \mathrm{~Hz}$ to $2 \mathrm{kHz}$ ) through a Digitimer D150 Amplifier (Digitimer Ltd., Welwyn Garden City, Herts, UK), stored at a sampling frequency of $5 \mathrm{kHz}$ on a personal computer for off-line analysis (SigAvg Software, Cambridge Electronic Design, Cambridge, UK). The sweep time ranged from $-20 \mathrm{~ms}$ up to $100 \mathrm{~ms}$ following stimulus onset. During the experiment, the EMG activity was continuously monitored with visual feedback (oscilloscope) and sound (speakers). Patients were asked to activate the sternocleidomastoid muscles by trying to elevate the head of about 30 degrees from the horizontal plan. We registered 200 tracks, first rectified and then averaged, and thus measured the mean P13N23 amplitude of the ipsilateral sternocleidomastoid muscle.

\subsection{Electrophysiological parameters: VEP recording}

We registered the VEP contextually to cVEMPs recording, in analogy to Todd's work (Todd et al., 2003), in which a small P10-N15 biphasic wave was recorded from $\mathrm{Cz}$ (referred to $\mathrm{Fz}$, according to the 10-20 international system). This component was also registered in profoundly deaf subjects, together with the cVEMPs, thus suggesting a vestibular origin (Rosengren et al., 2006). We used standard EEG disk electrodes. Skin-electrode impedance was kept $\leq 5 \mathrm{k} \Omega$. The time-analysis was set at $100 \mathrm{~ms}$, with a $3 \mathrm{~Hz}-$ $2 \mathrm{kHz}$ notched band-pass. We averaged 200 trials for the main cortical VEP wave and thus measured the mean P10-N15 amplitude.

\subsection{Electrophysiological parameters: TMS cortical excitability measures}

In order to assess sensory-motor long-term potentiation-like changes induced by the VT, we tested the motor cortex excitability following PAS at $\mathrm{T}_{0}$ and $\mathrm{T}_{1}$, as measure of induced cortical spike-timing dependent plasticity, in analogy to a previous work on lower limbs (Jayaram et al., 1998). We delivered 120 pairs of stimuli at a frequency of $0.5 \mathrm{~Hz}$, continuously for 4 minutes. Each pair of stimuli consisted of a conditioning electrical stimulus, applied on the common peroneal nerve of the paretic lower limb, distally and anteriorly to the fibula head, at $120 \%$ of motor threshold (i.e. the minimum current level that induced a visible and palpable twitch in tibialis anterior), and the magnetic test stimulus, delivered after the conditioning stimulus, at an individual inter-stimulus interval of $8 \mathrm{~ms}$ from the individual motor evoked potential -MEPlatency, at $90 \%$ of the resting motor threshold, applied on the hot-spot for the tibialis anterior muscle in the affected hemisphere. The magnetic stimuli was delivered through a standard eight-shaped coil, connected to a Magstim Rapid Stimulator (Magstim Company, Whitland, Dyfed, UK), with average diameters of the loops of the coil of $9 \mathrm{~cm}$, with a biphasic-wave magnetic pulse, and an amplitude of about $300 \mu \mathrm{s}$. The coil was oriented backwards and parallel to the midline, approximately $2 \mathrm{~cm}$ posterior to the vertex, on the optimal site on the scalp to get the wider MEP amplitude from the target tibialis anterior muscle. The current in the coil flowed in the direction of the handle during the first phase of the stimulus, and thus had a 
postero-anterior direction within the cortex. The electric conditioning stimulus was delivered through a Digitimer D160 Stimulator (Digitimer, Welwyn Garden City, Herts, UK), with the cathode proximally located. The stimulus had a square-wave morphology with $1 \mathrm{~ms}$ of duration. MEPs were obtained through magnetic monophasic stimuli delivered by a highpower Magstim_200 Stimulator (Magstim, Whitland, Dyfed, UK). The coil was placed on the tibialis anterior muscle hot-spot. The rise time of the magnetic monophasic stimulus was about $100 \mu \mathrm{s}$ with a to-zero of about $800 \mu \mathrm{s}$. The current flowed in handle direction during the rise-time of the magnetic field, thus with a posterior-anterior direction. We preliminarily evaluated the resting motor threshold, defined as the smallest stimulus intensity able to evoke a peak-topeak MEP of $50 \mathrm{uV}$ in tibialis anterior muscle at rest, in at least five-out-ten tracks consecutively (Rossini et al., 2004). Then, we applied an intensity of stimulation of $130 \%$ of resting motor threshold. EMG was recorded through $\mathrm{Ag}-\mathrm{AgCl}$ surface electrodes applied over the tibialis anterior muscle using a classic muscle belly-tendon montage. Signals were amplified and filtered (from $32 \mathrm{~Hz}$ to $1 \mathrm{KHz}$ ) through a Digitimer D150 Amplifier (Digitimer Ltd., Welwyn Garden City, Herts, UK), and stored using a sampling frequency of $10 \mathrm{KHz}$ on a personal computer for off-line analysis (Signal Software, Cambridge Electronic Design, Cambridge, UK).

\subsection{Statistical methods}

The Kruskal-Wallis test was used to test the homogeneity between the two groups before the study. In case of significant inter-group differences at baseline, the variables were included as covariates in the model. We applied the Wilcoxon signed-ranks test on each $\mathrm{T}_{1}-\mathrm{T}_{0}$ outcome measure at intra-group level. The Mann-Whitney U-test was used to evaluate the $\left(\mathrm{T}_{1}-\mathrm{T}_{0}\right) \%$ inter-group differences. The Bonferroni correction was used for multiple comparisons. Two-tailed $p$-values $<0.05$ were considered significant. A Fisher's test was carried out to assess the correlation between clinical and electrophysiological parameters. Values are reported as mean \pm standard deviation (sd).

\subsection{Ethical aspects}

The experimental protocol was approved by the Local Ethics Committee. Written informed consent was provided by all the participants.

\section{Results}

\subsection{Baseline data}

At baseline, multiple independent-sample KruskalWallis tests showed that demographic and clinical characteristics were not statistically different between the two groups $(p>0.2)$ (Table 1).

\subsection{Primary outcomes}

Patients well-tolerated either the pVT or the rVT. Indeed, no patients reported any side effect that led to protocol withdrawal or any kind of discomfort during the ERIGO sessions. More in detail, the VAS score was always $\leq 6$ in both groups, either during or after each VT session, whereas MBP, HR, and $\mathrm{pO}_{2}$ varied by $\pm 10 \%$ during the VT training. There were no significant differences in $\mathrm{BP}, \mathrm{HR}$, and $\mathrm{pO}_{2}$ improvement in the two groups. Instead, G1 showed a higher degree of amelioration in the remaining parameters in comparison to G2. Moreover, only the G1 group showed an improvement of RCPM, FM, and PASS scores. More detailed data are reported in Table 2.

\subsection{Secondary outcomes}

Although there were significant PAS after-effects in both the groups, the improvement in cortical plasticity was more evident in G1 than G2. Moreover, G1 showed a higher degree of amelioration in cVEP and cVEMPS amplitude in comparison to G2. More detailed data are reported in Table 2 .

\subsection{Clinical-electrophysiological correlations}

rVT correlation analysis showed strong PAS after-effects/RCPM $(Z=2.5, p=0.009)$, PAS aftereffects/FM $(Z=2.1, p=0.04)$, and VEMPs/PASS $(\mathrm{Z}=2.8, p=0.006)$ correlations.

\section{Discussion}

One of the main goals of post-stroke physical rehabilitation consists in the achievement of postural control and arousal's improvement (Wahl and Schwab, 2014). Rehabilitation on a tilt-table has been reported to be a useful way to mobilize severely impaired or non-cooperating patients, since it improves circulation, 
Table 2

Shows the intra- and inter-group effects (respectively upper and lower grid) of VT on cognitive, electrophysiological, tolerance, and clinical parameters (percentual or mean values \pm s.d.) from baseline $-\mathrm{T}_{0}$ - to one-month therapy $-\mathrm{T}_{1}$. NS stands for non-significant

\begin{tabular}{|c|c|c|c|c|c|c|c|c|}
\hline & & & \multicolumn{3}{|c|}{ G1 } & \multicolumn{3}{|c|}{$\mathrm{G} 2$} \\
\hline & & & $\mathrm{T}_{0}$ & $\mathrm{~T}_{1}$ & $\mathrm{Z}, \mathrm{p}$ & $\mathrm{T}_{0}$ & $\mathrm{~T}_{1}$ & $\mathrm{Z}, \mathrm{p}$ \\
\hline \multirow[t]{8}{*}{ Primary outcomes } & Cognitive domain & RCPM & $17 \pm 3$ & $33 \pm 13$ & $2.4,0.02$ & $19 \pm 6$ & $26 \pm 16$ & NS \\
\hline & Verticalization tolerance & VAS & $5 \pm 1$ & $2 \pm 1$ & $-2.5,0.01$ & $4 \pm 1$ & $2 \pm 1$ & $2,0.04$ \\
\hline & & $\mathrm{BP}(\mathrm{mmHg})$ & $113 \pm 3$ & $101 \pm 6$ & $-2.3,0.01$ & $116 \pm 6$ & $105 \pm 9$ & $-2.3,0.01$ \\
\hline & & HR (bpm) & $81 \pm 5$ & $76 \pm 9$ & $-1.9,0.04$ & $90 \pm 6$ & $79 \pm 16$ & $-2.1,0.03$ \\
\hline & & $\mathrm{pO}_{2}(\%)$ & $98 \pm 2$ & $99 \pm 3$ & NS & $97 \pm 5$ & $98 \pm 6$ & NS \\
\hline & Clinical parameters & MRC & $2 \pm 1$ & $4 \pm 1$ & $2.5,0.01$ & $2 \pm 3$ & $3 \pm 2$ & $2,0.04$ \\
\hline & & FM & $13 \pm 3$ & $25 \pm 6$ & $2.2,0.03$ & $12 \pm 6$ & $19 \pm 7$ & NS \\
\hline & & PASS & $3 \pm 1$ & $8 \pm 3$ & $2.3,0.01$ & $3 \pm 3$ & $5 \pm 3$ & NS \\
\hline \multirow[t]{5}{*}{ Secondary outcomes } & $\begin{array}{c}\text { Electrophysiological } \\
\text { parameters }\end{array}$ & post-PAS MEP (mV) & $0.5 \pm 0.3$ & $0.9 \pm 0.3$ & $2,0.04$ & $0.5 \pm 0.4$ & $0.6 \pm 0.4$ & NS \\
\hline & & $\mathrm{cVEP}(\mu \mathrm{V})$ & $2 \pm 0.2$ & $5 \pm 0.5$ & $2.4,0.02$ & $2 \pm 0.3$ & $4 \pm 0.2$ & $22,0.04$ \\
\hline & & $\operatorname{cVEMPS}(\mu \mathrm{V})$ & $12 \pm 2$ & $23 \pm 5$ & $2.3,0.01$ & $16 \pm 5$ & $20 \pm 3$ & $2,0.04$ \\
\hline & & & G1 & $\mathrm{G} 2$ & G1 vs. G2 & & & \\
\hline & & & $\% \mathrm{~T}_{1}-\mathrm{T}_{0}$ & $\% \mathrm{~T}_{1}-\mathrm{T}_{0}$ & $\mathrm{Z}, \mathrm{p}$ & & & \\
\hline \multirow[t]{8}{*}{ Primary outcomes } & Cognitive domain & RCPM & $+16 \pm 10$ & $+7 \pm 10$ & $2.2,0.03$ & & & \\
\hline & Verticalization tolerance & VAS & $-60 \pm 10$ & $-50 \pm 10$ & $2,0.04$ & & & \\
\hline & & $\mathrm{BP}(\mathrm{mmHg})$ & $-12 \pm 3$ & $-11 \pm 3$ & NS & & & \\
\hline & & HR (bpm) & $-5 \pm 4$ & $-11 \pm 10$ & NS & & & \\
\hline & & $\mathrm{pO}_{2}(\%)$ & $+1 \pm 1$ & $+1 \pm 1$ & NS & & & \\
\hline & Clinical parameters & $\mathrm{MRC}$ & $+2 \pm 1$ & $+1 \pm 1$ & $2.2,0.03$ & & & \\
\hline & & FM & $+92 \pm 10$ & $+58 \pm 7$ & $2.7,0.008$ & & & \\
\hline & & PASS & $+166 \pm 30$ & $+66 \pm 2$ & $2.7,0.008$ & & & \\
\hline \multirow{4}{*}{$\begin{array}{l}\text { Secondary } \\
\text { outcomes }\end{array}$} & Electrophysiological parameters & & & & & & & \\
\hline & & post-PAS MEP (mV) & $0.4 \pm 0.1$ & $0,1 \pm 0.1$ & $3.7,<0.001$ & & & \\
\hline & & $\operatorname{cVEP}(\mu \mathrm{V})$ & $3 \pm 0.3$ & $2 \pm 0.1$ & $2.4,0.02$ & & & \\
\hline & & cVEMPS $(\mu \mathrm{V})$ & $11 \pm 3$ & $4 \pm 2$ & $2.4,0.02$ & & & \\
\hline
\end{tabular}

RCPM: raven colored progressive matrices; BP: blood pressure; HR: heart rate; MRC: muscle research council; FM: Fugl-Meyer; PASS: Postural Assessment Scale for Stroke patients; cVEP: cortical vestibular evoked potentials; cVEMPs: cervical vestibular evoked myogenic potentials; PAS paired associative stimulation; MEP: motor evoked potential.

prevents contractures, and increases pulmonary ventilation (Chang et al., 2004; Horn et al., 2005; Cumming et al., 2011). Our pilot-study supports the safety and effectiveness of ERIGO verticalization in bedridden post-stroke patients, even in the sub-acute phase. In fact, we found a greater improvement in nearly all clinical and safety outcomes after rVT, with particular regard to FM (lower limb motor function), PASS (postural control), and RCPM (cognitive abilities). Such better post-rVT improvement could be related to the fact that robotic rehabilitation may offer standardized, intensive and repetitive exercises, a proper body weight support, with an appropriate sensory feedback amount and a controlled progressive verticalization.

Notably, robot-based rehabilitation has been shown to improve motor performance by boosting brain plasticity (Pellegrino et al., 2012; Duret et al., 2014; Basteris et al., 2014).

To this end, the novelty of our study consists in the electrophysiological evaluation of vestibular system and of sensory-motor cortex plasticity after a prolonged ERIGO training, which, to the best of our knowledge, has never been performed so far. Although vestibular system excitability improved in both the groups, the sensory-motor plasticity was more significantly induced in G1. Interestingly, the electrophysiological ameliorations we observed were related to PASS, RCPM, and FM improvements, thus suggesting the pivotal role of plasticity modification in enhancing postural control, lower limbs functions, global cognitive functions and thus reducing the patient's functional impairment (Kleim and Jones, 2008). Moreover, the stronger sensory-motor and vestibular system plasticity improvement in G1 could have been also promoted by the FES (Hooper, 2010). In fact, the combination of progressive verticalization with FES-cycling leg movements may offer a great amount of sensory feedback may enhance cortical plasticity, as during the normal walking (Jerin and Gürkov, 2014; Daikuya et al., 2003; Wei et al., 1998; Kuznetsov et al., 2013). 
Another interesting data in our study concerns the hemodynamic effects of rVT, which improved the mean BP and the HR, although similarly to pVT. It has been shown that robotic tilt-tables are effective in preventing blood pressure drops (Kuznetsov et al., 2013), since stepping movements, together with the FES vascular effects (Chi et al., 2008; Luther et al., 2008), may prevent orthostatic responses to verticalization by improving the venous return and, hence, potentiate the cardiac output and the cerebral blood flow. Despite we didn't directly assess the cerebral blood flow, its increase in specific brain regions has been observed in chronic stroke patients after constraint therapy, leading to an indirect evidence of treatment-related cortical reorganization (Sawaki et al., 2008). Analogously, we may argue that a greater cerebral blood flow modulation during rVT in comparison to pVT could further support plastic changes within sensory-motor areas and vestibular system, with the consequent motor and cognitive function amelioration (Raethjen et al., 2008; Do et al., 2011; Wieser et al., 2010; Reinstrup et al., 1994; Duncan and Owen, 2000).

\section{Conclusion and future perspectives}

In conclusion, ERIGO device could be, in our opinion, a safe rehabilitative strategy to restore motor system and cognitive functions, and thus to avoid a chronic condition thanks to its potential in improving functional plasticity within sensory-motor and vestibular systems. Nevertheless, larger sample size trials are needed in order to consolidate our preliminary finding and help move our promising neurophysiological approach closer to clinical practice for a more reliable evaluation of rVT after-effects.

The authors declare neither conflicts of interest nor financial support.

\section{References}

American Physical Therapy, Association, (1997). Guide to Physical Therapist Practice. Physical Therapy, 77, 1168-1650.

Andersson, G., Hagman, J., Talianzadeh, R., Svedberg, A., \& Larsen, H.C. (2003). Dual-Task Study of Cognitive and Postural Interference in Patients with Vestibular Disorders. Otol Neurotol, 24, 289-293.

Baltz, M., Lietz, H.L., Trott-Sausser, I., Kalpakjian, C., \& Brown, D. (2013). Tolerance of a tilt table protocol in an in-patient stroke unit setting: A pilot study. J Neurol phys ther, 37, 9-13.

Basteris, A., Nijenhuis, S.M., Stienen, A.H., Buurke, J.H., Prange, G.B., \& Amirabdollahian, F. (2014). Training modalities in robot-mediated upper limb rehabilitation in stroke: A framework for classification based on a systematic review. J Neuroeng Rehabil, 11, 111.

Benaim, C., Pérennou, D.A., Villy, J., Rousseaux, M., \& Pelissier, J.Y. (1999). Validation of a standardized assessment of postural control in stroke patients: The Postural Assessment Scale for Stroke Patients (PASS). Stroke, 30, 1862-1868.

Bogataj, U., Gros, N., Kljajic, M., Acimovic, R., \& Malezic, M. (1995). The rehabilitation of gait in patients with hemiplegia: A comparison between conventional therapy and multichannel functional electrical stimulation therapy. Phys Ther, 75, 490502 .

Chang, A.T., Boots, R., Hodges, P.W., \& Paratz, J. (2004). Standing with assistance of a tilt table in intensive care: A survey of Australian physiotherapy practice. Aust J Physiother, 50, 51-54.

Chang, W.H., \& Kim, Y.H. (2013). Robot-assisted Therapy in Stroke Rehabilitation. J Stroke, 15, 174-181.

Chi, L., Masani, K., Miyatani, M., Adam Thrasher, T., Wayne Johnston, K., Mardimae, A., Kessler, C., Fisher, J.A., \& Popovic, M.R. (2008). Cardiovascular response to functional electrical stimulation and dynamic tilt table therapy to improve orthostatic tolerance. J Electromyogr Kines, 18, 900-907.

Colebatch, G., Halmagyi, G.M., \& Skuse, N.F. (1994). Myogenic potentials generated by a click-evoked vestibulocollic reflex. JNNP, 57, 190-197.

Cullen, K.E., \& Roy, J.E. (2004). Signal processing in the vestibular system during active versus passive head movements. J Neurophysiol, 91, 1919-1933.

Cumming, T.B., Thrift, A.G., Collier, J.M., Churilov, L., Dewey, H.M., Donnan, G.A., \& Bernhardt, J. (2011). Very early mobilization after stroke fast-tracks return to walking: Further results from the phase II AVERT randomized controlled trial. Stroke, 42, 153-158.

Daikuya, S., Nishimori, T., Tanino, Y., Takasaki, K., \& Suzuki, T. (2003). Test-retest reliability for recording the Erb's point potential with a change of recording posture. Electromyogr Clin Neurophysiol, 43, 273-275.

Do, A.H., Wang, P.T., King, C.E., Abiri, A., \& Nenadic, Z. (2011). Brain computer interface controlled functional electrical stimulation system for ankle movement. J Neuro Eng Rehab, 8, 49.

Duncan, J., \& Owen, A.M. (2000). Common regions of the human frontal lobe recruited by diverse cognitive demands. Trends Neurosci, 23, 475-483.

Duret, C., Courtial, O., Grosmaire, A.G., \& Hutin, E. (2014). Use of a robotic device for the rehabilitation of severe upper limb paresis in subacute stroke: Exploration of patient/robot interactions and the motor recovery process, BioMed Research International, in press.

Ferrante, S., Pedrocchi, A., Ferrigno, G., \& Molteni, F. (2008). Cycling induced by functional electrical stimulation improve the muscular strength and the motor control of individuals with post-acute stroke. Eur J PhysRehabil Med, 44, 159-167.

Gladstone, J.D., Danells, C.J., \& Black, S.E. (2002). The FuglMeyer Assessment of Motor Recovery after Stroke: A Critical Review of Its Measurement. Neurorehabil Neural Repair, 16, 232. 
Goldberg, J.M., Smith, C.E., \& Fernández, C. (1984). Relation between discharge regularity and responses to externally applied galvanic currents in vestibular nerve afferents of the squirrel monkey. J Neurophysiol, 51, 1236-1256.

Gordon, N.F., Gulanick, M., Costa, F., Fletcher, G., Franklin, B.A., Roth, E.J., \& Shephard, T. (2004). Physical Activity and Exercise Recommendations for Stroke Survivors. Circulation, 109, 2031-2041.

Hathout, G., Nayak, N., Abdulla, A., \& Huang, J. (2014). The revised sonographic NASCET index: A new hemodynamic parameter for the assessment of internal carotid artery stenosis, Ultraschall Med, Epub ahead of print.

Hooper, S.L. (2010). Central Pattern Generators. UK: Encyclopedia of Life Sciences, John Wiley \& Sons.

Hummelsheim, H., \& Mauritz, K.H. (1993). The neurophysiological basis of exercise physical therapy in patients with central hemiparesis. Fortschr Neurol Psychiatr, 61, 208-216.

Jayaram, G., \& Stinear, J.W. (1998). Contralesional paired associative stimulation increases paretic lower limb motor excitability post-stroke. Exp Brain Res, 185, 563-570.

Jerin, C., \& Gürkov, R. (2014). Posture-induced changes of ocular vestibular evoked myogenic potentials suggest a modulation by intracranial pressure. Exp Brain Res, 232, 2273-2279.

Kaplan, R.M., \& Saccuzzo, D.P. (2009). Standardized tests in education, civil service, and the military, Psychological testing, Principles, applications, and issues. Belmont, CA: Wadsworth.

Kim, J., \& Curthoys, I.S. (2004). Responses of primary vestibular neurons to galvanic vestibular stimulation (GVS) in the anaesthetized guinea pig. Brain Res Bull, 64, 265-271.

Kleim, J.A., \& Jones, T.A. (2008). Principles of ExperienceDependent Neural Plasticity: Implications for Rehabilitation After Brain Damage. J Speech Lang Hear R, 51, 225-239.

Kuznetsov, A.N., Rybalko, N.V., Daminov, V.D., \& Luft, A.R. (2013). Early post-stroke rehabilitation using a robotic tilt-table stepper and functional electrical stimulation. Stroke Res Treat 2013, 94605-94606.

Luther, M.S., Krewer, C., Müller, F., \& Koenen, A. (2008). Comparison of orthostatic reactions of patients still unconscious within the first three months of brain injury on a tilt table with and without integrated stepping. A prospective, randomized crossover pilot trial. Clin Rehab, 22, 1034-1041.

Medical Research, Council, (1981). Aids to the examination of the peripheral nervous system. Memorandum no. 45; London: Her Majesty's Stationery Office.

Pellegrino, G., Tomasevic, L., Tombini, M., Assenza, G., Bravi, M., Sterzi, S., Giacobbe, V., Zollo, L., Guglielmelli, E., Cavallo, G., Vernieri, F., \& Tecchio, F. (2012). Inter-hemispheric coupling changes associate with motor improvements after robotic stroke rehabilitation. Restor Neurol Neurosci, 30(6), 497-510.

Peszczynski, M., Benson, D.F., Collins, J.M., Darley, F.L., Diller, L., Greenhouse, A.H., Katzen, F.P., Lake, L.F., Rothberg, J.S., \& Waggoner, R.W. (1972). II. Stroke rehabilitation. Report of the joint committee for stroke facilities. Stroke, 3, 375-407.

Pittaccio, S., Zappasodi, F., Tamburro, G., Viscuso, S., Marzetti, L., Garavaglia, L., Tecchio, F., \& Pizzella, V. (2013). Passive ankle dorsiflexion by an automated device and the reactivity of the motor cortical network. Conf Proc IEEE Eng Med Biol Soc 2013, 6353-6356.

Pollock, A., Baer, G., Campbell, P., Choo, P.L., Forster, A., Morris, J., Pomeroy, V.M., \& Langhorne, P. (22). (2014). Physical rehabilitation approaches for the recovery of function and mobility following stroke. Cochrane Database Syst Rev, 4, CD001920.

Popovic, D.B., Sinkaer, T., \& Popovic, M.B. (2009). Electrical stimulation as a means for achieving recovery of function in stroke patients. NeuroRehabilitation, 25, 45-58.

Precht, W. (1979). Vestibular mechanisms. Annu Rev Neurosci, 2 , 265-289.

Raethjen, J., Govindan, R., Binder, S., Zeuner, K.E., Deuschl, G. \& Stolze, H. (2008). Cortical representation of rhythmic foot movements. Brain Res, 1236, 79-84.

Raven, J., Raven, J.C., \& Court, J.H. (2004). Manual for Raven's Progressive Matrices and Vocabulary Scales, Harcourt Assessment, San Antonio. TX.

Reinstrup, P., Ryding, E., Algotsson, L., Berntman, L., \& Uski, T. (1994). Effects of nitrous oxide on human regional cerebral blood flow and isolated pial arteries. Anesthesiology, 81, 396402 .

Risey, J., \& Briner, W. (1990). Dyscalculia in Patients with Vertigo. J Vestib Res, 1, 31-37.

Rosengren, S.M., \& Colebatch, J.G. (2006). Vestibular evoked potentials (VsEPs) in patients with severe to profound bilateral hearing loss. Clin Neurophysiol, 117, 1145-1153.

Rosengren, S.M., Govender, S., \& Colebatch, J.G. (2011). Ocular and cervical vestibular evoked myogenic potentials produced by air- and bone-conducted stimuli: Comparative properties and effects of age. Clin Neurophysiol, 122, 2282-2289.

Rossini, P.M., Barker, A.T., Berardelli, A., Caramia, M.D., Caruso, G., Cracco, R.Q., Dimitrijević, M.R., Hallett, M., Katayama, Y., Lücking, C.H., Maertens de Noordhout, A.L., Marsden, C.D., Murray, N.M.F., Rothwell, J.C., Swash, M., \& Tomberg, C. (1994). Non-invasive electrical and magnetic stimulation of the brain, spinal cord and roots: Basic principles and procedures for routine clinical application. Report of an IFCN committee. Electroencephalogr Clin Neurophysiol, 91, 79-92.

Sawaki, L., Butler, A.J., Leng, X., Wassenaar, P.A., Mohammad, Y.M., Blanton, S., Sathian, K., Nichols-Larsen, D.S., Wolf, S.L., Good, D.C., \& Wittenberg, G.F. (2008). Constraint-Induced Movement Therapy Results in Increased Motor Map Area in Subjects 3 to 9 Months After Stroke. Neurorehabil Neural Repair, 22, 505-513.

Stefan, K., Kunesch, E., Cohen, L.G., Benecke, R., \& Classen, J. (2000). Induction of plasticity in the human motor cortex by paired associative stimulation. Brain, 123, $572-584$.

Todd, N.P., Rosengren, S.M., \& Colebatch, J.G. (2003). A short latency vestibular evoked potential (VsEP) produced by boneconducted acoustic stimulation. J Acoust Soc Am, 114, 32643272 .

Tong, M.F., Li, L.S., \& So, E.F. (2006). Gait training of patients after stroke using an electromechanical gait trainer combined with simultaneous functional electrical stimulation. Phys Ther, 86, 1282-1294. 
Uchino, Y., Sato, H., Kushiro, K., Zakir, M., Imagawa, M., Ogawa, Y., Katsuta, M., \& Isu, N. (1999). Cross-striolar and commissural inhibition in the otolith system. Ann N Y Acad Sci, 871, 162-172.

Vallar, G., Bottini, G., Rusconi, M.L., \& Sterzi, R. (1993). Exploring somatosensory hemineglect by vestibular stimulation. Brain, 116, 71-86.

Vallar, G., Sterzi, R., Bottini, G., Cappa, S., \& Rusconi, M.L. (1990). Temporary remission of left hemi-anesthesia after vestibular stimulation. A sensory neglect phenomenon. Cortex, 26, 123131.

Van der Wees, P.J., \& Mead, J. (2004). Framework for Clinical Guideline Development in Physiotherapy. Brussels (Belgium): European Region of World Confederation for Physical Therapy.

Verheyden, G., Nieuwboer, A., Feys, H., Thijs, V., Vaes, K., \& De Weerdt, W. (2005). Discriminant ability of the Trunk Impairment Scale: A comparison between stroke patients and healthy individuals. Disabil Rehabil, 27, 1023-1028.

Vitenzon, A.S., Mironov, E.M., \& Petrushanskaya, K.A. (2005). Functional electro-stimulation of muscles as a method for restoring motor functions. Neurosci Behav Physiol, 35, 709-714.

Wahl, A.S., \& Schwab, M.E. (2014). Finding an optimal rehabilitation paradigm after stroke: Enhancing fiber growth and training of the brain at the right moment. Front Hum Neurosci, 8, 381 .
Wei, J., Zhao, L., Yan, G., Chen, W., Ren, W., \& Duan, R. (1998). Dynamic change of ERPs related to selective attention to signals from left and right visual field during head-down tilt. Acta Astronaut, 42, 323-330.

Wieser, M., Haefeli, J., Bütler, L., Jäncke, L., Riener, R., \& Koeneke, S. (2010). Temporal and spatial pat-terns of cortical activation during assisted lower limb movement. Exp Brain Res, 203, 181-191.

Yan, T., Hui-Chan, C.W.Y., \& Li, S.L.W. (2005). Functional electrical stimulation improves motor recovery of the lower extremity and walking ability of subjects with first acute stroke. Stroke, $36,80-85$.

Yardley, L., Gardner, M., Bronstein, A., Davies, R., Buckwell, D., \& Luxon, L. (2001). Interference between postural control and mental task performance in patients with vestibular disorder and healthy controls. JNNP, 71, 48-52.

Young, J., \& Forster, A. (2007). Review of stroke rehabilitation. $B M J, 334,86-90$.

Zhou, G. and Cox, L.C. Vestibular evoked myogenic potentials: History and overview. Am J Audiol, 13, 135-143. 